\title{
Las condiciones asociadas a la experiencia de éxito universitario de las mujeres con discapacidad
}

\section{Conditions associated with the successful university experience of women with disabilities}

\author{
Marcela Ramírez Morera \\ Universidad de Costa Rica, San José, Costa Rica \\ marcela.ramirez@ucr.ac.cr \\ https://orcid.org/0000-0002-5498-5656
}

\section{Resumen}

Introducción: En el presente artículo $^{1}$ se expone el proceso y resultado desarrollado en una investigación doctoral que analiza las condiciones de éxito académico de las mujeres con discapacidad en la educación superior costarricense. En esta se muestra que ellas son mujeres determinadas, con metas claras y que han transitado a través de trayectorias académicas caracterizadas por el enfrentamiento constante de barreras y el apoyo incondicional de las familias como eje fundamental de su éxito.

Objetivo: El artículo comprende las experiencias de éxito académico de las mujeres en situación de discapacidad en las universidades costarricenses, a partir de las condiciones endógenas, familiares y sociales.

Método: Se acude al paradigma de la codificación de la teoría fundamentada, mediante la escucha atenta de los textos discursivos obtenidos de las narraciones biográficas, desarrolladas por medio de cuatro focos de discusión (personal, familiar, docente y grupo de pares).

Resultados: Entre los principales hallazgos se destacan que: la mayoría han estudiado en el ámbito de las ciencias sociales, residen en el Gran Área Metropolitana o migraron a esta, en búsqueda mayores apoyos educativos. Se caracterizan por ser mujeres determinadas y establecerse metas claras, son lideresas, empáticas y activistas; se encuentran en edades productivas, sus padres y madres finalizaron solamente la primaria, y desde sus inicios educativos recibieron los ajustes razonables. Las familias han sido facilitadoras en todas las etapas formativas, destacando el protagonismo de las madres. El profesorado más cercano a estas ha recibido procesos de capacitación en el ámbito de derechos humanos, accesibilidad y discapacidad. Asimismo, los grupos de pares muestran relaciones respetuosas entre los semejantes más cercanos, destacan la interacción con las mujeres en situación de

\footnotetext{
${ }^{1}$ Parte de este artículo se presentó en la Jornada de Investigación de la Escuela de Trabajo Social en el año 2021 y en el Congreso Internacional sobre Discapacidad y Universidad organizado por la Fundación Once en ese mismo año.
} 
discapacidad como medio de aprendizaje, lo que configuró las experiencias de éxito académico.

Conclusiones: Entre las principales conclusiones se identifica que la mayoría de mujeres participantes realizaron sus estudios universitarios en carreras de ciencias sociales y educación. La mayoría de sus estudios se realizaron en el área metropolitana debido a que en esta zona existe una oferte más amplia de servicios de apoyos y servicios institucionales. Dichos apoyos educativos durante la trayectoria académica fueron un factor que influyó positivamente en el éxito de las mujeres con discapacidad, Sin embargo, se reconoce que las mujeres accedían a los espacios educativos enfrentaban barreras actitudinales, lo cual las llevo a demandar sus derechos educativos.

Palabras Claves: Éxito académico, Mujeres con discapacidad, Educación superior, Teoría fundamentada, Accesibilidad.

\section{Abstract}

Introduction: This article presents the process and result developed in a doctoral research that analyzes the conditions of academic success of women with disabilities in Costa Rican higher education, where it is shown that they are specific women, with clear goals and that have traveled through academic trajectories characterized by the constant confrontation of barriers and the unconditional support of families as a fundamental axis of their success. Objective: The article covers the experiences of academic success of women with disabilities in Costa Rican universities, based on endogenous, family and social conditions.

Method: We turn to the paradigm of the codification of Grounded Theory, by listening attentively to the discursive texts obtained from biographical narratives, developed through four foci of discussion (personal, family, teacher and peer group).

Results: Among the main findings are that: most have studied in the field of social sciences, reside in the Greater Metropolitan Area or migrated to it, seeking greater educational support. They are characterized by being determined women and setting clear goals, are leaders, empathetic and activists, they are at productive ages, their parents finished only primary school, and from their educational beginnings they received reasonable accommodations. Families have been facilitators in all the formative stages, highlighting the role of mothers. Teachers closest to them have received training in the areas of human rights, accessibility and disability. In addition, peer groups show respectful relationships among the closest peers, highlight the interaction with women with disabilities as a means of learning, which shaped the experiences of academic success.

Conclusions: Among the main conclusions, it is identified that the majority of women participants completed their university studies in social sciences and education. Most of his studies were carried out in the metropolitan area because in this area there is a wider offer of support services and institutional services. These educational supports during the academic 
trajectory were a factor that positively influenced the success of women with disabilities, However, it is recognized that women accessed educational spaces faced attitudinal barriers, which led them to demand their educational rights.

Keywords: Academic success, Women with disabilities, Higher education, Gounded theory, Accessibility.

\section{Introducción}

El objetivo del presente artículo es comprender la experiencia de éxito académico desde las propias voces de las mujeres con discapacidad, visualizando sus condiciones académicas, motivaciones familiares para ingresar a la universidad, apoyo recibido por docentes, compañeros y compañeras de clase, incluso la concepción que ellas mismas tienen de su situación de discapacidad y realidad académica. Se busca reflexionar sobre las condiciones que han permitido el éxito académico en la educación superior de mujeres con dicha situación, con la intención de promover la recurrencia de tales eventos y recursos, para el mejoramiento de la participación con equidad de las mujeres con discapacidad en el entorno universitario. Entendiendo por experiencia de éxito académico, tal y como Moriña y Melero (2016) mencionan, el acceso a la universidad, la permanencia y la finalización de los estudios universitarios.

Entre las aportaciones más relevantes de las autorías referente a la temática de estudio, se cuenta con las siguientes consideraciones. Calvo-Babbio, Muñoz-Cantero y Novo-Corti (2015), Lehtomäki, Matonya y Trotta, (2015), Samudio y Edda (2016) y Erten (s.f) estudian históricamente la presencia de mujeres en la educación superior en Europa, Norteamérica y en algunos países centroamericanos. Entre los resultados más sobresalientes de estos estudios se destaca lo siguiente: la presencia tímida de las mujeres en la educación superior, la carencia de capacitación del personal administrativo y docente sobre la discapacidad, se visualiza la discapacidad como limitación para el éxito académico, la invisibilización de las necesidades individuales, las escasas medidas de formación de actitudes favorables hacia la discapacidad desde la infancia. Y algunos factores de éxito académico se refieren al apoyo del entorno familiar, formación permanente del profesorado $\mathrm{y}$ asistencia financiera.

La mayoría de las investigaciones según Marcela (2021) enfatizan que el éxito académico se basa en el ingreso, permanencia y conclusión de los estudios universitarios. Sin embargo, dicha área del éxito académico, esta mediada por factores externos como la motivación del núcleo familiar y el profesorado de primaria y secundaria, la accesibilidad en el entorno universitario, tanto arquitectónicamente como en los procesos de admisión, ingreso, permanencia y conclusión de los estudios; así como en el apoyo financiero para acceder a la educación superior.

Por otra parte, entre los factores endógenos de las personas con éxito académico en los estudios se encuentran: la motivación, el autoconcepto, el planteamiento de metas, el 
estado de ánimo, la perseverancia y la resiliencia, los cuales se vinculan al apoyo familiar y docente. Sobre este aspecto, las investigaciones evidencian que entre las barreras que afectan el éxito académico están la percepción de discapacidad como limitación para el éxito académico, la tímida participación histórica de las mujeres en la educación superior y, en particular, de las mujeres en situación de discapacidad. Aunado a esto, algunas universidades continúan reproduciendo acciones asistencialistas y rehabilitadoras en el momento de brindar servicios y apoyos al estudiantado con discapacidad. (Ramírez 2021)

Se evidencia que ninguno de los estudios toma en cuenta como sujetos de investigación a las familias, al profesorado ni al personal administrativo vinculados con el proceso educativo de las mujeres en situación de discapacidad, y la necesidad de comprender el éxito académico como una experiencia y vivencia de las estudiantes universitarias con discapacidad.

Asimismo, entre los hallazgos más sobresalientes, se destaca que solo una de las investigaciones retoma a las mujeres estudiantes con discapacidad en los contextos universitarios; y que la mayoría de los estudios se inclina hacia el análisis y discusión de los servicios de apoyo universitario para la población con discapacidad en general, es decir, hombres y mujeres.

Por lo tanto, se afirma que el género y la discapacidad interactúan, colocando a las personas en una posición desigual. El género por su parte se comprende como una construcción sociocultural, fundamentada en las diferencias biológicas y la a discapacidad como una elaboración social y restricción en la participación, por ende, son características que socialmente se traducen en desigualdad y discriminación, las cuales son reproducidas en las instituciones con discursos androcéntricos, como sucede en las entidades de educación superior (Ramírez 2011).

Aunque el género y la discapacidad se asumen desde la vulnerabilidad, es necesario plantear un nuevo orden a través de la participación social y la visualización de las mujeres con discapacidad como protagonistas de su propio éxito, particularmente del éxito académico en la educación superior. Por lo tanto, la argumentación anterior visualizaba un aporte novedoso y significativo en el ámbito de los estudios interdisciplinarios en discapacidad.

\section{Objetivo}

\section{Diseño y método}

Comprender las condiciones académicas asociadas a las experiencias de éxito de las mujeres con discapacidad en la educación superior en Costa Rica.

\section{Metodología}

El objeto de estudio de la investigación se refiere a las experiencias del éxito académico de las mujeres con discapacidad, estudiantes universitarias. Se acude al enfoque cualitativo (Taylor y Bogdan 1987, Levitt 2018). El proceso seguido para establecer la 
fidelidad al tema estudiado se concreta en el acercamiento al diálogo discursivo de las personas entrevistadas, con una adaptación de la teoría fundamentada (Glaser y Strauss 1967). La recopilación de datos se realiza a través de narraciones biográficas y, además, se recurre a elementos transductivos (Ibánez 1985 y Ferreira 2010). La estrategia de análisis se apoya en el estudio comparado de casos (Yin 1994; Coller 2005), adecuado a la metodología cualitativa (Stake 1995).

Cabe destacar que, de las 88 entrevistas, de acuerdo con el muestreo teórico, se codificaron 40 narraciones biográficas de 10 casos, cuyo procesamiento de la información se realizó con el programa Atlas. Ti 8. Según Glaser y Strauss (1967), el muestreo teórico es una estrategia de selección secuencial vinculada con la interpretación de los datos en la investigación, se genera en el seno de la teoría fundamentada y tiene como objetivo identificar la información.

Según Strauss y Corbin (2002), la recolección de los datos es la reducción intencionada a través de un instrumento, en particular las narraciones biográficas de la realidad tan compleja y extensa del tema desarrollado, con el fin de hacerla más comprensible y focalizada. Por tanto, se codificaron 40 biografías, las cuales se agruparon en «casos». Cada uno de ellos está configurado por las cuatro unidades analíticas; es decir, mujer y hombre en situación de discapacidad, persona de apoyo, docente universitario y compañero o compañera.

Con el fin de acercarse al fenómeno estudiado, se recurre al diseño del modelo analítico, el cual está constituido por las cuatro dimensiones analizadas en la investigación, estas se encuentran configuradas por: la dimensión personal, que se caracteriza por las condiciones sociodemográficas, endógenas e itinerarios académicos de las personas interactuantes en la indagación (personas con discapacidad); la dimensión familiar y el grupo de pares, que comparten a su vez aspectos sociodemográficos y endógenos, más actitudes familiares y políticas educativas, entre otras. Finalmente, la cuarta refiere a la dimensión docente, compuesta por las condiciones de accesibilidad y los apoyos educativos brindados en los contextos universitarios. Las cuatro dimensiones dialogan entre sí sobre la vida universitaria, procesos académicos y la convivencia sociofamiliar.

\section{Trabajo de campo y análisis de datos}

La investigación está configurada a través de varios momentos complementarios, comprensivos y circulares. Se inició con la delimitación del objeto de estudio y las preguntas de investigación, las cuales llevaron a elaborar líneas orientadoras metodológicas.

El segundo momento inicia entre junio del 2018 y abril del 2019 con el trabajo de campo, el cual siguió una planificación establecida para acercarse a la información y realizar las entrevistas biográficas, según los focos discursivos y el proceso desarrollado mediante el paradigma de la codificación. 
Durante los meses de setiembre del 2019 y marzo del 2020 se desarrolló el tercer momento, caracterizado por la triangulación teórica con el método de la comparación de casos constante (Yin 1994 y Coller 2005). Este se realiza mediante un análisis de los discursos presentados en las entrevistas biográficas ejecutadas tanto en las pruebas piloto como en las narraciones utilizadas en la investigación.

El cuarto momento se extendió entre los meses de abril y noviembre del año 2020, el cual consiste en la revisión teórica, construcción de los resultados y redacción del documento final.

\section{Resultados}

En el presente apartado se realizará un análisis relacional con el objetivo de desarrollar y elaborar vinculaciones de los datos a partir de la interacción entre las cuatro unidades de observación (personas con discapacidad, familiares, grupo de pares y docentes) de las experiencias de éxito académico. Las interacciones relacionales se ejecutan a partir de los vínculos entre códigos, atributos y grupos teóricos, originadas en la codificación abierta, cuyo resultado fue las categorías explicativas.

\section{La presencia de la caracterización endógena en el éxito académico}

En esta relación se observa la interacción dinámica entre las categorías teóricas: caracterización endógena y la perspectiva de éxito académico. Específicamente los códigos y atributos relacionales con las características endógenas son: metas claras, realización personal, familiar y profesional, autonomía e independencia y la permanente actitud para derribar barreras.

En cuanto al código de liderazgo, sobresale el atributo de realización personal y profesional. En la perspectiva de éxito académico se encuentran los elementos del desempeño profesional, búsqueda de equilibrio, insistencia, determinación y ejercicio en el ámbito laboral.

Se evidencia una relación clara entre el atributo de realización personal familiar y profesional con el desempeño en la profesión, el cual es protagonista en el éxito académico. De igual manera, dicha interacción se vincula con la búsqueda de equilibrio personal, emocional y social. En esta misma línea, se encuentran la autonomía y la independencia, las cuales influyen en la insistencia y determinación presentes en la perspectiva de éxito académico. Esta última también se vincula con la permanente actitud por derribar barreras.

Por consiguiente, la realización personal y profesional interactúan con el ámbito laboral. Es evidente que, en la caracterización endógena, la insistencia, metas claras y liderazgo son códigos intervinientes en el concepto de éxito académico, vinculado a la satisfacción con la carrera elegida, actitud personal y la obtención de títulos universitarios.

De acuerdo con los horizontes argumentativos citados anteriormente, estos conducen a la comprensión del concepto que se tiene del éxito académico de las mujeres con 
discapacidad, el cual está vinculado con la satisfacción de la carrera seleccionada y la actitud personal. Por lo tanto, se evidencia que las mujeres mantienen una actitud de liderazgo, lo cual las lleva a ser insistentes y determinadas en lo que se proponen, dispuestas a derribar las barreras que se les imponen, acontecimiento que contribuye a una realización personal y a un desempeño profesional óptimo. Además, se observa una satisfacción con la carrera que cursaron o que se encuentran desarrollando.

Se observa que la autonomía y la independencia se ubican como variables interdependientes del éxito académico.

\section{El protagonismo del contexto familiar en el concepto de éxito académico}

Otra relación originada a partir de la codificación axial es la que se refiere al protagonismo de la familia en el éxito académico, lo cual contribuye a la permanencia y finalización de la formación académica. Aquí interviene la naturalización de la situación de la discapacidad en el entorno familiar, realización personal, insistencia y determinación, empoderamiento y reconocimiento de los derechos de la persona con discapacidad por parte de la familia, protagonismo y determinación de las madres para que sus hijos e hijas alcancen la realización personal mediante sus aspiraciones y expectativas y el deseo de superación de las madres.

Por lo tanto, se hipotetiza que el contexto familiar contribuye a que las estudiantes con discapacidad permanezcan y finalicen sus trayectorias académicas exitosamente. El papel de las madres es significativo en dichos procesos, según se nota desde los primeros años de vida y durante los diferentes ciclos del desarrollo humano.

Además, son evidentes las manifestaciones de violencia hacia las madres y estudiantes a través de la imposición de las barreras. Por ello, se deduce que, en dichos espacios académicos, las personas identifican y reconocen con mayor criticidad esta inconcebible realidad.

En relación con el código proyectos de vida, específicamente la característica de realización personal y profesional, el desempeño y la satisfacción en ambos ámbitos caracterizan el concepto de éxito académico vinculado con la identificación de la carrera seleccionada. Finalmente, dentro de este mismo código se rescatan los apoyos necesarios, los cuales se relacionan con los ajustes institucionales presentes en el ingreso a la universidad.

En la presente categoría explicativa, se reitera la participación fundamental de la familia en el éxito académico, mediante la naturalización de la discapacidad, el empoderamiento y reconocimiento de los derechos de las personas, situación que contribuye al desarrollo de los proyectos de vida, vinculados con las áreas personales y profesionales.

\section{Interacciones entre dinámicas de grupo de pares presentes en el éxito académico}

En las interacciones entre las dinámicas del grupo de pares y su participación en el éxito académico, se encuentran relaciones entre los apoyos incondicionales brindados por 
colegas, la permanencia y la finalización de la formación académica. Por lo tanto, se observa que el apoyo permanente entre grupo de pares contribuye a obtener calificaciones y a sobrellevar las demandas académicas, característica que facilita el cumplimiento y la conclusión de los estudios universitarios.

El código de concientización del grupo de pares se identifica mediante la actitud colaborativa, la cohesión grupal y el trabajo en equipo, además se encuentra relacionado con el construir saberes, la afinidad por la carrera seleccionada y el cumplimiento y cierre de los estudios. En esta misma línea se ubica la participación en actividades académicas, el cual interactúa con el concepto de éxito académico vinculado a la satisfacción personal. De acuerdo con esta categoría, se puede interpretar que las relaciones positivas y asertivas entre colegas contribuyen a la permanencia en los procesos formativos universitarios.

De acuerdo con el concepto de éxito académico, desde la perspectiva de cumplimiento de metas y la actitud personal, interactúa el aprendizaje adquirido por los grupos de pares, particularmente en lo relacionado con la insistencia, determinación, aspiraciones y expectativas. El compañerismo se caracteriza por el apoyo de los grupos de pares, actitud de acogida y naturalización de las situaciones de discapacidad. Estas características se vinculan con el cumplimiento, finalización de estudios, actitud docente y asumir los retos, dichos atributos constituyen el éxito académico desde la óptica de metas, actitud personal e ingreso a la universidad. Por lo tanto, se puede concluir que, el acompañamiento y la presencia del grupo de pares es un factor facilitador en el éxito académico de la persona con discapacidad.

\section{La accesibilidad: dimensión interviniente en el éxito académico}

Con el objetivo de analizar la participación de las diferentes instancias universitarias que brindan servicios y recursos de apoyo en las experiencias de éxito académico de las mujeres en situación de discapacidad, se procede a relacionar la categoría teórica de la dimensionalidad de la accesibilidad con la perspectiva de éxito académico. Particularmente, en la dimensión actitudinal el código de concientización docente concurre con la disposición del profesorado, el cumplimiento y la finalización de los estudios formativos.

En esta misma línea, el atributo de búsqueda de apoyo por parte de las personas docentes se interseca con la construcción de saberes, disposición docente, cumplimiento, conclusión de estudios universitarios y apoyos institucionales significativos; códigos y atributos que caracterizan el concepto de éxito académico desde la perspectiva de la satisfacción con la carrera seleccionada y las metas establecidas.

Continuando con el argumento anterior, la cuota de capacitación está interactuando con los atributos de disposición docente, cumplimiento, conclusión de estudios, apoyos institucionales significativos, permanencia y finalización de la formación académica. En relación con el concepto de éxito académico desde la óptica de actitud personal y 
establecimiento de metas, esta se encuentra vinculada con la dimensión actitudinal, particularmente con los atributos de confianza, apertura y disposición docente.

Los apoyos institucionales significativos se conceptualizan a partir de la preparación académica y profesional desde la perspectiva de la discapacidad y las experiencias previas del profesorado, además de la disposición docente, la capacitación en temas de discapacidad y educación inclusiva (apoyos educativos), la cual facilita la permanencia y la finalización de los estudios universitarios.

La accesibilidad como dimensión interviniente de un contexto que configura el éxito académico visualiza los apoyos educativos como las personas facilitadoras, el uso de la tecnología y el espacio físico accesible, los cuales interactúan con códigos y atributos como el cumplimiento, conclusión de estudios y apoyos institucionales significativos, reconocimiento de servicios universitarios que brindan asesoría y acompañamiento a la población estudiantil con discapacidad, acontecimientos que se convierten en elementos promotores del éxito académico.

En la presente categoría explicativa se identificaron tanto apoyos como factores limitantes para las personas con discapacidad, entre los códigos y atributos con mayor concurrencia se encuentran los obstáculos actitudinales, vinculados con estereotipos en discapacidad y género, actitudes estereotipadas del profesorado, discriminación, barreras en espacios físicos, exclusión educativa y violencia.

Un hecho sobresaliente en esta dimensión explicativa consiste en que las instituciones focalizan la accesibilidad física, la cual genera barreras que limitan la permanencia en la educación superior de las personas con discapacidad. Según los textos interactuantes, las instituciones de educación superior han realizado esfuerzos significativos por la consolidación de una infraestructura más accesible. No obstante, aún existen barreras actitudinales que generan violencia estructural y simbólica.

Entre las conclusiones más sobresalientes de los resultados se tiene que las actitudes de liderazgo, insistencia y determinación son características imperantes de las mujeres con discapacidad. situación que las impulsa a derribar barreras constantemente. Asimismo, la participación de la familia se convierte en un eje fundamental para el éxito académico de las mujeres con discapacidad. Por otra parte, también se puede identificar como factor facilitador el acompañamiento y la complicidad entre el grupo de pares. Por último, las instituciones de educación superior han centralizado los esfuerzos en la accesibilidad física, sin embargo, esta dimensión no es la única que garantiza el éxito académico, ya que aún existen barreras actitudinales generadoras de violencia estructural y simbólica.

\section{Discusión y conclusiones}

Una vez finalizadas las codificaciones abiertas y la axial, se procede a efectuar la codificación selectiva. Para lo que se toman los cinco primeros códigos de mayor densidad, junto a las concurrencias entre códigos y atributos manifestados por las mujeres y hombres 
en situación de discapacidad, interactuantes de la investigación. Para ello, se recurrió a responder los objetivos específicos mediante el desarrollo de las conclusiones, las cuales se concretan a través de los ejes centrales de cada fin en particular. Por lo tanto, a continuación, se desarrollan distintos subapartados, donde cada uno responde a cada objetivo planteado para este artículo.

\section{La interacción de las características sociodemográficas de las mujeres con discapacidad en la educación superior costarricense}

La mayoría de las mujeres universitarias con discapacidad tienen edades de entre 25 y 58 años, su formación profesional es Derecho, Trabajo Social, Educación Especial, Orientación, Filología, Sociología, con maestrías en derechos humanos, discapacidad y educación. Ellas residen en el área metropolitana debido a la concentración de apoyos y servicios institucionales en la zona central del país. Incluso, en dos casos, las familias decidieron trasladarse de la zona rural hacia la urbana para que sus hijas contaran con mayores oportunidades educativas.

Las mujeres con discapacidad han finalizado sus estudios de grado universitario en Ciencias Sociales, se desempeñan laboralmente en este ámbito. Particularmente ellas trabajan en los campos de derechos humanos, discapacidad, Trabajo Social y docencia universitaria dentro de la institucionalidad pública, aunque algunas de ellas brindan también servicios privados.

La argumentación anterior coincide con los estudios efectuados por Alba et al (2012) y Fayez (2017), quienes refieren que la mayoría del estudiantado con discapacidad se matricula en carreras vinculadas con Ciencias Sociales; incluso, se menciona que alcanzan mayor éxito académico en dicho ámbito. No obstante, la autoría no argumenta las razones de esta situación.

Por lo tanto, se deduce que en esta área se identifican mayores premisas teóricas que contribuyen a comprender las situaciones de exclusión social y la necesidad de garantizar los derechos sociales, políticos y económicos a las distintas poblaciones.

En relación con el ámbito familiar, las madres y los padres han finalizado la primaria, solo un padre cuenta con formación universitaria. Ellos brindaron los apoyos educativos necesarios a sus hijas durante sus primeros años de vida. Las madres se dedicaron a labores domésticas, una de ellas combinaba dichas actividades con trabajos remunerados fuera del hogar. Los proyectos de vida de las madres están vinculados con la finalización de los estudios superiores de sus hijas, mantienen el deseo de que las estudiantes alcancen lo que se proponen, particularmente en los espacios educativos y laborales.

Los resultados evidenciaron las interseccionalidades analizadas por Kimberlé Crenshaw (1990), Ocampo (2016) y La Barbera (2016); estas autoras y autor visualizan que las mujeres residentes, tanto en el ámbito rural como urbano, mantienen una actitud persistente de alcanzar sus metas establecidas, particularmente académicas. Además, es 
evidente en ellas la constante búsqueda de oportunidades educativas. Para lograrlo, algunas de las familias se trasladaron al área metropolitana.

La mayoría de las mujeres se encuentra en edades económicamente productivas, y aquellas con hijas e hijos han tenido atrasos para finalizar sus estudios de postgrado. Solo una de ellas está cursando los estudios doctorales, no tiene hijos e hijas ni pareja.

La mayoría de las mujeres pertenece a la clase baja y media, presentan discapacidades físicas y visuales, y en algún momento de su vida han vivido violencia de género, estructural, simbólica y doméstica (Bourdieu 1994; Ocampo 2013; Hernández 2014; Pérez-Castro 2019).

Dada la complejidad de las características presentadas por las participantes, la perspectiva interseccional utilizada para el análisis en esta investigación permitió ver las interacciones dadas entre las características de las argumentaciones anteriores y que ya habían sido señaladas por diversas personas autoras. Por ejemplo, Pérez-Castro (2019) cita que entre las mujeres con discapacidad es posible detectar los siguientes tipos de violencia: violencia interpersonal y estructural, explotación y abuso sexual o económico a través de la mendicidad, esterilizaciones forzadas, aislamiento, segregación, falta de acceso a la información, rechazo a sus derechos familiares, negación de accesos a la justicia, violaciones a su libertad, falta de acceso a la educación, salud, trabajo, vivienda o a la administración de sus propios bienes, entre otros.

Es evidente que, al momento de dialogar con la revisión teórica, se concluye que mediante las experiencias de éxito académico de las mujeres con discapacidad es necesario acudir a las configuraciones emancipadoras, transductivas e interseccionales, con el fin de comprender y acercarse a la cotidianidad; en donde los escenarios sociodemográficos que actúan entre sí conforman una complejidad que no es posible comprender si se estudia cada circunstancia por separado.

\section{Condiciones académicas que facilitan o limitan el éxito, dinámica entre características sociodemográficas de las mujeres con discapacidad}

Los apoyos educativos que requirieron las estudiantes en los trayectos formativos iniciales fueron suministrados por las familias, las cuales al mismo tiempo recibieron empoderamiento de servicios de apoyo bajo la modalidad de educación especial. Sin embargo, es de suma importancia aclarar que las mujeres accedían a los espacios educativos enfrentando barreras actitudinales, por lo que se vieron obligadas a acudir a la exigibilidad de derechos educativos, la cual vivenciaron mediante el uso de los ajustes razonables educativos que les fueron provistos.

La argumentación anterior coincide con lo que plantean Said Rücker (2011), Palma et al (2016) y Druckemiller et al (2015) sobre la participación de la familia en los entornos educativos, mediante el apoyo permanente a sus hijas, con el objetivo de acceder a mejores condiciones de vida. En relación con la caracterización endógena de los discursos interactuantes, en el estudio se observa una relación directa entre metas claras y 
determinación, particularmente en las mujeres con discapacidad. Se puede deducir que si la persona es determinada tiene mayores oportunidades y posibilidades de alcanzar sus metas.

La determinación también se evidencia en las madres, quienes tienen como objetivo la formación académica de sus hijas. Por ejemplo, en el código de metas claras, tres mujeres con discapacidad mencionan la determinación, en cambio entre los hombres ninguno la cita. Por lo tanto, se observa una relación clara entre caracterización endógena y el contexto familiar, particularmente entre los códigos, metas claras y determinación, tanto entre las mujeres con discapacidad como en sus madres.

El horizonte argumentativo anterior coincide con lo desarrollado por Heiman (2006), Kennett et al (2009), Karimi (2012) y López y Polo (2015), quienes mencionan que entre los factores intrínsecos de las personas con éxito académico participantes en los diferentes estudios realizados por ellos se encuentran la motivación, el autoconcepto, el planteamiento de metas y el estado de ánimo, los cuales están vinculados con el apoyo familiar y docente; además se muestran otras características personales como la perseverancia y resiliencia.

Asimismo, empatía, liderazgo y activismo son características endógenas que sobresalen en las mujeres, situación que contribuye a desarrollar relaciones asertivas dentro del grupo de pares; peculiaridad que está ligada a otro código significativo en las estudiantes, el cual se refiere al interés por las demás personas. Por ejemplo, el 50\% de las mujeres y el $0 \%$ de los hombres de los grupos de pares mencionan la importancia de las relaciones de amistad. En específico el código de liderazgo y el atributo de capacidad de implicación y reacción es referido por el $50 \%$ de las mujeres y el $0 \%$ de los hombres con discapacidad.

De acuerdo con el resultado anterior, Espiñeira-Bellón (2013) señala la importancia de que las relaciones personales garanticen entornos favorables por parte de los compañeros y compañeras del estudiantado con discapacidad mediante la concientización. Además, este autor agrega que es necesario el compromiso de la comunidad, familia, liderazgo democrático, servicios y recursos de apoyo, para evitar la discriminación y garantizar la capacitación y el diseño de cursos de acuerdo con las distintas discapacidades con las que se interactúa en los contextos universitarios.

Aunado a lo anterior, la mayoría de las personas docentes refiere haber desarrollado experiencias en diversos ámbitos de participación social, particularmente en aquellos espacios relacionados con los derechos humanos. Por lo tanto, se puede inferir que dichas vivencias generan concientización, lo que permite mayor implicación y disposición en brindar apoyos educativos a las mujeres con discapacidad y se convierte en un factor de éxito académico para las estudiantes.

Al respecto, con el resultado anterior, Durán-Aponte y Durán-García (2015) mencionan que el desempeño docente está vinculado con las oportunidades de permanencia que brinda la entidad universitaria al estudiantado, lo que, al mismo tiempo, va a influir en la inclusión de la población estudiantil. Por ejemplo, las características de personalidad 
actitudinales y socioafectivas pueden resultar en experiencias académicas con posibilidad de crear relaciones que motiven al estudiantado para alcanzar el éxito académico.

De igual manera, se observa que las mujeres con discapacidad desarrollan más estrategias para enfrentar situaciones diversas y tienen mayor capacidad de adaptación en comparación con los hombres. Dicha caracterización es reconocida por el profesorado y por los grupos de pares de ellas. Aunado a eso, se observa una relación directa entre la búsqueda de apoyos y la identificación de soluciones que ellas mantienen en sus realidades cotidianas para conseguir las metas que se proponen a lo largo de sus trayectorias académicas.

\section{Condiciones académicas, familiares y sociales que configuran el éxito académico}

Como se evidenció tanto en la codificación abierta y axial como en la selectiva, la familia como facilitadora es un factor determinante en las experiencias de éxito académico de las mujeres con discapacidad. Particularmente, en este código concurren los atributos de la implicación incondicional de la familia y promoción de la autonomía e independencia, los cuales son reconocidos tanto por mujeres como por hombres en situación de discapacidad. Además, los atributos de naturalización de la situación de la discapacidad en el entorno familiar, el apoyo educativo para continuar las trayectorias académicas, empoderamiento, asumir papeles específicos para brindar apoyo y el reconocimiento de la importancia de los núcleos familiares primarios son referidos solamente por las mujeres con discapacidad.

Los resultados destacados en el párrafo anterior coinciden con lo que citan DuránAponte y Elvira-Valdés (2015), quienes mencionan a Roca-Cuberes (2012), Saldaña y Barriga (2010) y Donoso y Schiefelbein (2007), personas autoras que recurren a la teoría de la persistencia de Tinto $(1975,1997)$ para proponer un modelo de integración, enfatizando en la influencia de los antecedentes familiares y en la escolaridad previa sobre las intenciones y metas del estudiantado. Una vez que la persona inicia la universidad las situaciones de los grupos primarios y educativos impactarán en los resultados académicos y en el comportamiento social, al mismo tiempo, en la manera en que afrontan los acontecimientos cotidianos en el contexto universitario, lo cual contribuye a que la persona tenga mayor posibilidad de continuar con los estudios superiores.

De acuerdo con las argumentaciones anteriores, se infiere que visualizar a las familias como facilitadoras es más significativo para las mujeres que para los hombres con discapacidad. Por lo tanto, se convierte en elemento interviniente en las experiencias de éxito académico de las mujeres. Hasta el momento, este resultado no se ha identificado en investigaciones involucradas con la presente temática de análisis.

Los códigos de madre como protagonista y el proceso de ajuste de la discapacidad son mayormente reconocidos por familiares mujeres de los casos 1,2,4,5 y por las mujeres y los hombres con discapacidad de los casos 1, 5 y 8. Por lo que se hipotetiza que las familias se observan como parte de las experiencias de éxito académico de las mujeres con discapacidad, sin dejar de lado el transitar vivido. En relación con lo anterior, Barrientos 
(2013) menciona que la participación de las madres y los padres en los procesos educativos garantiza calidad y mejoramiento del rendimiento académico. Además, esa participación genera autoconfianza y acceso a la información para con el centro educativo.

De acuerdo con las líneas anteriores, Acuña et al (2017) mencionan la importancia de que las madres comprendan la situación de discapacidad de sus hijos e hijas para brindar apoyos y acompañamientos necesarios en las diversas trayectorias académicas, acontecimientos que se evidencian en los resultados de esta investigación.

En los casos 1, 2, 4 y 5 particularmente, las mujeres con discapacidad refirieron al código de la sobreprotección, caracterizado por la transferencia del cuido a otras personas, la vigilancia permanente, la reproducción de la sobreprotección y la presencia de esta en los grupos fraternales. Por lo que se interpreta que las experiencias de éxito académico de las mujeres han sido mediatizadas por la sobreprotección; es decir, ellas se han visto obligadas a enfrentar y desarrollar estrategias que derrumben el muro de esta forma de violencia sutil implicada en la sobreprotección.

En esta misma línea, Domínguez y Vásquez (2016) refieren que los factores de la sobreprotección se extienden desde confundir el amor que le dan a su hijo o hija hasta facilitarles y proporcionar todo en su vida, limitándoles su autonomía y autocontrol. Además, quienes ejercen mayor sobreprotección son las madres, debido a que ellas se sienten culpables, tienen miedo a las críticas, mantienen sentimientos de lástima y creen que la persona con discapacidad no podría continuar sus ciclos vitales sin el apoyo de ellas, elementos impuestos por un sistema patriarcal. En la presente investigación se observó que las mujeres, a pesar de experimentar esta manifestación de violencia, continuaron con sus trayectorias académicas, de forma que deconstruyeron la situación de la discapacidad y la condición de género, mediante la actitud permanente en derribar barreras presentadas en los diferentes recorridos educativos, ya que ellas deseaban alcanzar con determinación las metas propuestas.

A partir de la codificación abierta y axial, se evidencia que el código de proyecto de vida cuenta con mayor cantidad de citas asociadas, entre las cuales la realización personal y profesional son referidas en mayor cantidad por los hombres, particularmente los casos 1, 3 y 4 y solo el $50 \%$ de las mujeres hacen referencia a este, casos 7 y 8 . Además, el atributo de proyecto de vida al servicio de otros solo es referido por una mujer y no por hombres. Por lo tanto, se hipotetiza que las mujeres aún mantienen rastros de las imposiciones patriarcales, las cuales constituyen un velo que limita las miradas hacia otros horizontes de crecimiento y mejoramiento de sus calidades de vida.

La argumentación anterior se vincula con lo destacado por Martínez (2008), quien refiere que los estudios de los cuidados en los entornos familiares se han organizado en tres ámbitos: el primero de ellos se centra en el análisis de las actividades trabajadas en el área doméstica. El segundo se focaliza en el análisis interrelacionar entre los trabajos desarrollados en el campo doméstico-familiar y profesional. Y, finalmente, el tercero enfatiza 
en la especificidad de los cuidados, situación que refuerza que las mujeres continúan siendo las encargadas del cuidado de otras personas integrantes de la familia, principalmente de aquellas en situación de discapacidad y personas adultas mayores.

Por lo tanto, se presenta para ellas una sobrecarga con grandes costos emocionales y profesionales, particularmente en aquellas generaciones «pinza» (Martínez 2008), las cuales surgen cuando las mujeres cuidan a sus padres, madres y a otras personas que requieren de apoyos permanentes, resultando en una doble exigencia para aquellas mujeres que laboran fuera de los contextos familiares y que deben tener una multipresencia.

Martínez (2008) continúa manifestando la necesidad de deconstruir la «naturalización» del ser y del deber ser de la adjudicación de los cuidados por parte de las mujeres, los cuales son confundidos con la feminidad; así como de eliminar la falacia de que los cuidados son brindados con amor, dinero, o cualquier otro intercambio de recursos materiales o simbólicos. No obstante, la mujer interactuante en la investigación que menciona la importancia de su proyecto de vida al servicio de otros responde a las premisas imperantes del patriarcado y a las concepciones de cuido existentes; por consiguiente, reproduce y trasciende los parámetros que caracterizan el género y la discapacidad, debido a que socialmente las mujeres con discapacidad se consideran frágiles.

En relación con el grupo de pares y el código concientización de este colectivo, las mujeres enfatizan más en la participación de actividades académicas; en cambio los hombres no lo mencionan. De lo que se deduce que la participación no es tema de interés para los hombres, elemento correlacional con las características endógenas de las mujeres en situación de discapacidad, entre las cuales sobresalen el liderazgo y participación activista en diferentes ámbitos sociales; aspecto que también se convierte en un hito histórico de las experiencias de éxito académico.

En esta misma línea, el código de colaboración entre el grupo de pares y trabajo en equipo, en específico el aprendizaje del grupo de pares, las mujeres lo reconocen más que los hombres, ya que ninguno de ellos lo refieren. Los resultados anteriores coinciden con lo estudiado por Palma et al (2016), cuando manifiestan que existen buenas y cordiales relaciones entre pares, mayor aceptación y aprendizaje mutuo, respeto, inclusión e interés para brindar los apoyos requeridos dentro de los contextos universitarios. Sin embargo, estos autores mencionan la existencia de cierta incomodidad por parte de las compañeras y compañeros en la cercanía corporal en el momento de requerir el apoyo para el traslado $\mathrm{u}$ otras actividades con implicación de mayores apoyos físicos. Además, se reconoce desde los grupos de pares que algunos ajustes razonables llegan a ser ciertos «privilegios».

Por consiguiente, dichas autorías reconocen la importancia de los apoyos por parte del profesorado y pares, con el fin de facilitar los procesos académicos y que el estudiantado con discapacidad se sienta comprendido y aceptado por el contexto inmediato que le rodea. Esto logra el acompañamiento necesario para la ejecución de ciertas actividades, beneficiando relaciones sociales entre el estudiantado con discapacidad y sin ella. 
Al respecto, las líneas argumentativas establecidas por Calvo-Babio, Muñoz-Cantero y Novo-Corti (2014) destacan que las actitudes del grupo de pares están vinculadas con la capacitación previa y experiencias cercanas con personas en situación de discapacidad.

\section{La conceptualización de éxito académico a partir de las experiencias de las mujeres con discapacidad}

El concepto de éxito académico destacado en los discursos se encuentra relacionado con la satisfacción personal y el desempeño profesional, particularmente las mujeres lo vinculan con la posibilidad de desarrollarse en los ámbitos laborales donde se pueda ejercer la carrera universitaria estudiada, y lo identifican con la construcción de saberes y la afinidad con la carrera, atributos que ningún hombre menciona.

Aunado a lo anterior, las mujeres vinculan el éxito académico con los códigos de metas, actitud personal y el atributo de insistencia y determinación. En cambio, los hombres solo lo relacionan con búsqueda de equilibrio (personal-emocional-social), aspiraciones y expectativas. En el momento de analizar los textos discursivos relacionados con el concepto de éxito académico desde las demandas educativas, solo un hombre lo observa mediante el atributo de cumplimiento y conclusión de estudios y ninguna de las mujeres lo visualiza desde este atributo.

Dicha argumentación mantiene un hilo común desarrollado por Durán-Aponte y Elvira-Valdés (2015), quienes mencionan a teóricos como Roca-Cuberes (2012), Saldaña y Barriga (2010) y Donoso y Schiefelbein (2007), quienes recurren a la teoría de la persistencia de Tinto $(1975,1997)$, la cual propone un modelo de integración donde los antecedentes familiares y la escolaridad previa influyen en las intenciones y metas del estudiantado.

La temática del ingreso a la universidad sobresale en el momento de consultar sobre la conceptualización del éxito académico, donde mujeres y hombres refieren por igual la importancia de los apoyos institucionales significativos.

De acuerdo con los apartados anteriores, los hallazgos de la investigación coinciden con lo estudiado por autores como Calvo-Babio, Muñoz-Cantero y Novo-Corti (2014), Lehtomäki, Matonya y Trotta (2015), Samudio (2016) y Erten (s.f), quienes destacan que la carencia de capacitación del personal docente sobre la discapacidad, visualizar esta situación y la invisibilización de las necesidades individuales se convierten en elementos que restringen las experiencias de éxito académico. En cambio, el apoyo del entorno familiar, particularmente de la madre, la formación permanente del profesorado y el apoyo institucional se convierten en elementos facilitadores de las experiencias académicas exitosas.

Un código emergente en la investigación es el que refiere a la violencia de género, particularmente hacia las madres y las mujeres con discapacidad, elemento que también fue identificado y estudiado por autores como Opini (2012), Expósito, Ferres y Megías (2013), Arvidsson, Staland-Nyman, Tideman y Widén (2016), Nicholas, Wang y Zhong (2017), 
quienes mencionan que la violencia dirigida a las mujeres con discapacidad está asociada a la situación socioeconómica, dependencia física, cargas familiares, bienestar emocional y a los estereotipos de género.

\section{La participación de las diferentes instancias universitarias en las experiencias de éxito académico}

En relación con las condiciones universitarias contextuales presentes en el éxito académico, se identifica el código de concientización docente, donde la búsqueda de apoyos por parte de la persona docente es reconocida por los hombres con discapacidad y los compromisos individuales, la confianza, disposición e incorporación de la perspectiva de la discapacidad son mencionados por las mujeres.

El argumento anterior se relaciona con lo que Durán-Aponte y Durán-García (2015) destacan acerca del desempeño docente, el cual está vinculado con las oportunidades de permanencia que brinda la entidad universitaria al estudiantado y que, por lo tanto, influye en la integración de la población estudiantil. Por ejemplo, la experticia y el dominio del profesorado sobre lo que enseña, sus competencias didácticas y pedagógicas, su capacidad para evaluar el desempeño del alumnado y las características de personalidad actitudinales y socioafectivas pueden resultar en experiencias académicas con posibilidad de crear relaciones que motiven al estudiantado para alcanzar el éxito académico. Dicha caracterización es reconocida por las mujeres con discapacidad mediante los diferentes fragmentos discursivos.

Los lineamientos anteriores también coinciden con los postulados planteados por el modelo de integración de Tinto (1985-1997), quien propone que la persistencia en la universidad es una manifestación del ajuste entre el estudiantado y la institución de educación superior, permitiéndole adquirir experiencias académicas y sociales que logran la permanencia con las interacciones positivas, entre iguales y el profesorado.

Por lo tanto, la experiencia de éxito académico va a estar mediatizada por el ajuste y las interacciones positivas entre docentes, estudiantes y la institución, los cuales están presentes en los procesos de ingreso, permanencia y finalización de estudios universitarios. Estos aspectos son elementos favorables para que las mujeres con discapacidad puedan experimentar trayectorias académicas exitosas.

Así mismo, se identificó en algunos casos la existencia de barreras actitudinales del profesorado, estas resistencias son mencionadas con mayor densidad por las mujeres.

Por otra parte, se evidencia que algunas universidades continúan reproduciendo acciones asistencialistas y rehabilitadoras en el momento de brindar servicios y apoyo al estudiantado con discapacidad, sin comprender ampliamente la diversidad que puede existir en la población usuaria.

En cuanto a los tipos de apoyo educativo que más mencionan las mujeres son las personas facilitadoras, el apoyo docente, las estrategias didácticas y evaluativas accesibles y 
la ampliación de la letra. Particularmente, en relación con las estrategias didácticas inclusivas, Gross Martínez (2020) destaca que estas deben visualizar elementos de comunicación con el grupo de estudiantes, con el fin de garantizar la participación de estos, además de presentar el contenido de aprendizaje vinculado con las características del alumnado, las barreras y la disciplina que se enseña. También, se debe concebir el material didáctico como facilitador de las actividades de aprendizaje desde la perspectiva del Diseño Universal para el Aprendizaje (DUA).

Por lo tanto, los ajustes razonables contribuyeron con las experiencias de éxito académico de las mujeres con discapacidad, elementos que se deben potencializar desde los servicios de apoyo universitario, con el fin de garantizar el derecho a la educación superior.

Las mujeres mencionan que la accesibilidad física es vital para su desarrollo académico. Además, reconocen que la falta de capacitación docente ocasiona violencia estructural y simbólica. En cambio, los hombres visualizan también la carencia de concientización del profesorado, pero no observan que esta situación desencadene los tipos de violencia citados anteriormente.

Los resultados anteriores coinciden con lo referido por Granada, Pomés y Sanhueza (2013), cuando afirman que la actitud del profesorado en la educación inclusiva puede ser tanto de facilitadores como de barreras en los procesos de enseñanza y aprendizaje. Por lo tanto, la implementación de acciones como planificación, colaboración y coordinación para atender las necesidades educativas garantiza el acceso a la educación. No obstante, dicha autoría señala que un docente con escasa formación inicial, falto de capacitación, poco tiempo de planificación y sin experiencia requerida en diversidad genera actitudes negativas.

En la dimensión de políticas, normas y procedimientos institucionales, particularmente en el código de barreras, el atributo que es más enfatizado por las mujeres es el de actitudes negativas hacia la discapacidad y la priorización en la participación. En relación con el último atributo señalado, se puede interpretar que las mujeres priorizan en cuáles actividades participar debido a los apoyos con los que cuentan para realizar las diferentes tareas académicas. Por ejemplo, si la comunidad universitaria desarrolla alguna acción recreativa o cultural y la mujer cuenta con el apoyo de una persona facilitadora, prefiere hacer uso de este ajuste para resolver las demandas académicas y no participar en actividades extracurriculares.

Finalmente, en relación con la categoría teórica, la perspectiva de la discapacidad y género, los códigos y atributos más mencionados por las mujeres con discapacidad son la concepción y el reconocimiento de la autonomía, las actitudes y oportunidades familiares, la centralización de la discapacidad, la lucha por el reconocimiento de los derechos y la naturalización de esta misma situación.

De acuerdo con la argumentación anterior, los códigos más relevantes referidos por las estudiantes dejan ver que ellas se observan a sí mismas como personas que solamente 
enfrentan una situación de discapacidad que es parte de su cotidianidad y no restringe su éxito académico.

\section{Marcos orientadores de los procesos formativos en el éxito académico de las mujeres con discapacidad}

Las ofertas formativas que se brindan en las diferentes instancias universitarias ejecutan programas y proyectos que permiten desarrollar el liderazgo de las mujeres con discapacidad que, desde horizontes investigativos, revelen el impacto de los parámetros patriarcales, con el fin de que ellas construyan diferentes realidades para el mejoramiento de su calidad de vida.

No obstante, las universidades estatales cuentan con políticas vinculadas al género, las cuales deberían incorporar la perspectiva de la discapacidad, con el objetivo de fortalecer lineamientos de éxito desde las aulas universitarias en las tres áreas sustantivas de las 5 universidades públicas. Particularmente, en las políticas de la Universidad de Costa Rica, en el eje VIII de Igualdad e Inclusividad, específicamente en las políticas 8.2.3 sobre género y 8.2.4 acerca de discapacidad, se destaca la que demanda «facilitar las condiciones de trabajo inclusivas y accesibles para personas en situación de discapacidad, diversidad sexual y etnia» (Consejo Universitario, 2020).

De igual manera, las políticas institucionales de la Universidad Técnica Nacional (UTN), citadas en el Plan Institucional de Desarrollo Estratégico Actualización 2018-2021 de la universidad, señalan la importancia de visualizar el género y la discapacidad en el quehacer universitario. Asimismo, el Instituto Tecnológico Nacional (TEC) cuenta con políticas institucionales en materia de discapacidad y políticas específicas para incrementar los niveles de equidad e igualdad en el ITCR, derivadas de las Políticas Generales 18 y 19, aprobadas en la sesión 2159 (2001), con el fin de garantizar el acceso del estudiantado que presente dichas interseccionalidades.

Adicionalmente, la Universidad Nacional de Costa Rica (UNA) incluye estas perspectivas en las Políticas Institucionales; con el fin de introducir los ejes de inclusión, discapacidad y equidad, aprobadas mediante el acta 2617 del año 2005. También, la universidad cuenta con la Política Institucional para el Empleo y la Inclusión de las Personas con Discapacidad (UNA-SCU-ACUE-027-2019) y políticas para la igualdad y equidad de género en la Universidad Nacional (UNA-SCU-ACUE-1071-2017).

Por último, se destacan las políticas universitarias implementadas por la Universidad Estatal a Distancia (UNED), mediante el documento Lineamientos de Política Institucional 2015-2019, el cual visualiza incluir los enfoques de discapacidad y género. Sin embargo, es necesario mencionar que dichas políticas se refieren al término de necesidades educativas especiales y no necesariamente al término discapacidad, hecho que evidencia la necesidad de actualizar las políticas mismas con el fin de garantizar los derechos humanos. 
A pesar de la existencia de diversas políticas institucionales, orientadas al género y a las personas con discapacidad, se evidencia que aún se mantiene la necesidad de promover en las diferentes mallas curriculares de las unidades académicas la incorporación de líneas investigativas, saberes pedagógicos y didácticos que construyan actitudes de liderazgo en las mujeres con discapacidad para su implicación sociopolítica y académica, permitiéndoles desempeñarse en agrupaciones tanto de reivindicación feminista como en el ámbito de discapacidad.

Además, los procesos formativos universitarios deben evitar centralizarse en las acciones eminentemente académicas. Se evidencia que las dinámicas universitarias se encuentran configuradas por una amplia gama de actividades culturales, deportivas y recreativas que son de suma importancia para el éxito académico y profesional de las mujeres con discapacidad. Si bien es cierto que ellas refieren que ocasionalmente buscan espacios de participación política y social en los escenarios accesibles, les gustaría desarrollar su activismo social en otros espacios de la universidad. Ellas no ejecutan estas actividades cotidianamente porque deben priorizar tareas académicas con el fin de permanecer y finalizar su carrera universitaria. Por ejemplo, es de suma importancia brindar un apoyo educativo como es el caso de las personas facilitadoras, no solo para la participación en los cursos formativos, sino en instancias tanto investigativas como culturales, deportivas, seminarios, coloquios, foros, entre otras; las cuales se convierten a la vez en formación académica y humanista, que son parte del quehacer universitario.

Otro aspecto significativo que emerge de la investigación consiste en que las instituciones de educación superior deben visualizar integralmente las condiciones de las mujeres, desde sus diferentes dimensionalidades humanas. No solo son estudiantes, sino académicas, investigadoras, activistas, lideresas, hijas, hermanas, tías, compañeras, amigas con historias desgarradoras de violencia generada por las diferentes interseccionalidades. Con lo anterior, es necesario la incorporación del enfoque de interseccionalidad en las políticas institucionales, proyectos y programas vinculados con el quehacer universitario, el cual permite la identificación de múltiples categorías que se encuentran entre sí.

Es de suma importancia que en los planes de estudio de todas las carreras universitarias se incorporen las perspectivas de género y discapacidad como ejes transversales a las diferentes áreas sustantivas que impactan la cotidianidad universitaria donde se generan acciones articuladas, inter e intrainstitucionales, que además potencialicen aspectos determinantes que construyen el éxito académico de las mujeres con discapacidad.

Un hallazgo significativo de la investigación fue la participación inminente de los grupos familiares en todas las trayectorias académicas de las mujeres con discapacidad, además de visualizarlas como personas, donde su situación de discapacidad es parte de su cotidianidad, lo cual no las restringe para cumplir sus metas académicas previamente establecidas, sino que más bien las impulsa. 
La argumentación anterior coincide con lo que Barrientos (2013) denomina el reajuste y adaptación de recursos, donde dicho grupo familiar, ejecuta modificaciones para brindar las respuestas necesarias que requieren las personas con discapacidad, lo cual garantizaría una «buena adaptación» que al mismo tiempo permita el éxito académico.

Si bien es cierto la presente investigación se realizó en un contexto donde las mujeres con discapacidad desarrollaron sus trayectorias académicas de forma exitosa desde la presencialidad, actualmente sería de gran relevancia científica indagar sobre las experiencias de éxito académico bajo la virtualidad y el uso de las tecnologías de comunicación e información; asimismo, acerca del requerimiento de ajustes razonables educativos vinculados con las herramientas digitales y las formas de comprender el ingreso, la permanencia y la finalización de las carreras universitarias.

Con el objetivo de evidenciar la importancia de garantizar el derecho a la educación superior desde las diferentes interseccionalidades, también se considera hallazgo de la presente investigación, la necesidad de dar respuesta a múltiples cuestionamientos como los siguientes: ¿cuáles necesidades de información y comunicación requiere una mujer con discapacidad que habita en una zona fronteriza?, ¿qué implicaciones de acceso tecnológico tiene una mujer migrante, indígena, afrodescendiente, residente en el área rural?, ¿cuáles ajustes razonables requiere una mujer con discapacidad visual que tiene poco acceso al internet o que debe utilizar plataformas virtuales no accesibles? Estas y otras interrogantes son de gran relevancia para futuras indagaciones y responden a los cambios sociales, políticos y económicos que se enfrentan actualmente.

De igual manera, es vital reflexionar y discutir acerca de las manifestaciones de violencia experimentadas por las mujeres con discapacidad y por sus madres en la presente época de confinamiento, lo que ha obligado a la virtualización de los procesos de enseñanza y aprendizaje, que puede generar tensiones detonantes de violencia en el contexto familiar debido a la carencia de un apoyo determinado, al aumento de los niveles de estrés y a la dificultad para continuar con sus estudios universitarios por esta vía.

El modelo metodológico desarrollado en la presente investigación contribuyó a la configuración de las experiencias de éxito académico a partir de la interacción de discursos, mediante las diversas dimensiones interactuantes en el ir y venir de la indagación. Esta situación permitió identificar elementos teóricos carentes en las publicaciones existentes sobre este horizonte investigativo. Un ejemplo de ello se hace evidente en los postulados conceptuales presentes en este texto, los cuales reflejan grandes vacíos en el momento de acercarse a las experiencias de éxito académico.

Por parte de los servicios de apoyo, es indispensable que promuevan las perspectivas interseccionales en su quehacer cotidiano. Aunque estas entidades fueron vitales en las experiencias de éxito académico de las mujeres con discapacidad, se evidencia la urgencia de evitar la focalización en la operativización de los servicios. Estos deben implicarse con el estudiantado más allá de atender los requerimientos oportunos, evolucionando y 
manteniéndose en constante actualización teórica, para acoplarse a las demandas actuales de las estudiantes y permitiendo el desarrollo en otras áreas de la vida universitaria, ya que las mujeres interactuantes en la investigación manifestaron la necesidad eminente de priorizar las demandas académicas con el objetivo de finalizar sus estudios.

La investigación evidencia el protagonismo de las familias en toda la trayectoria de éxito académico de las mujeres con discapacidad, mediante la implicación con sus hijas, generando espacios de autonomía e independencia. Por lo tanto, se recomienda que las familias continúen capacitándose, asesorándose y se encuentren anuentes al acompañamiento, lo cual permite el empoderamiento y la comprensión de la importancia de los procesos socioeducativos, claves en los diferentes ciclos vitales de las personas.

Parte del estudiantado manifestó la importancia de los grupos de pares en los diferentes procesos educativos, particularmente en el desarrollo de las tareas académicas, ya que en ocasiones implicaba complicidad para continuar con su formación universitaria. Con base en lo anterior, se recomienda a las compañeras y compañeros acercarse a sus colegas con discapacidad desde la perspectiva de pares, centralizándose en la condición de la persona como elemento principal y, posteriormente, en los apoyos brindados por su situación de discapacidad. Para ello es necesario que las instituciones de educación superior desarrollen estrategias que permitan la concientización dentro de las prácticas educativas ejecutadas por el profesorado universitario. Además de incluir, en los planes de estudio, cursos y saberes específicos vinculados con las realidades de la discapacidad y el género.

Se recomienda a las mujeres con discapacidad que tienen la oportunidad de continuar con estudios universitarios mantener una actitud determinante e insistente para alcanzar las diferentes metas personales, académicas y profesionales previamente establecidas. Además, se insta a desarrollar sus características de lideresas en los distintos espacios de participación social.

Apoyo financiero: No se contó con apoyo financiero de ninguna entidad para el desarrollo de esta investigación.

\section{Referencias}

Acuña-Arango, Lina María, Victoria Eugenia Cabrera-García, Diana Carolina MedinaCasallas y Flor Alba Lizarazo Sandoval. 2017. «Necesidades de la familia y de la escuela en la educación de niños y niñas con discapacidad intelectual». I+D Revista $\begin{array}{lllll}\text { de } & \text { Investigaciones, } & 9 & (1): & 126-137 .\end{array}$ https://www.udi.edu.co/revistainvestigaciones/index.php/ID/article/view/123

Pastor Alba, Carmen y Ainara Zubillaga. 2012. «La utilización de las TICs en la actividad académica de los estudiantes universitarios con discapacidad». Revista Complutense de Educación. $23 \quad$ (1): 23-50. Madrid. España. DOI: https://doi.org/10.5209/rev_RCED.2012.v23.n1.39100 
Arvidsson, Jessica, Carin Staland-Nyman, Magnus Tideman y Stephen Widén. 2016. «Postschool destination-A study of women and men with intellectual disability and the gender-segregated swedish labor market». Journal of Policy and Practice in Intellectual Disabilities, 13 (3): 217-226. DOI: https://doi.org/10.1111/jppi.12157

Oskarina, Palma, Camila,Barría, Ximena Lucero, Daniel Mella, Yaritza Santana, Enrique Seguel y Ximena Soto. 2016. «Estudio cualitativo del proceso de adaptación e inclusión de un grupo de estudiantes de educación superior con discapacidad». $\begin{array}{llll}\text { Magallania } & 44 & \text { (2): }\end{array}$ http://www.magallania.cl/index.php/magallania/article/view/873

Barrientos, Patricia. 2013. «La participación familia-escuela en México desde un enfoque inclusivo: reflexiones sobre familia y discapacidad». Revista Latinoamericana de $\begin{array}{llll}\text { Educación } & \text { Inclusiva } & 7 & \text { (2): }\end{array}$ http://repositoriocdpd.net:8080/bitstream/handle/123456789/1570/Art_BarrientosP LaparticipacionfamiliaescuelaenMexico_2014.pdf?sequence=1.

Bourdieu, Pierre. 1994. «La violenza simbólica. Entrevista Pierre Bourdieu». En la enciclopedia dell escienze filoso fiche de la RAI. http://www.emsf.rai.it/interviste.asp?d=388

Calvo-Babio, Nuria, Jesús-Miguel Muñoz-Cantero, e Isabel Novo-Corti. 2014. «Los futuros docentes y su actitud hacia la inclusión de personas con discapacidad. Una perspectiva de género». Anales de Psicología / Annals of Psychology, 31 (enero): 155-171. https://doi.org/10.6018/analesps.31.1.163631

Consejo Universitario Universidad de Costa Rica. (2020). «Políticas Institucionales 20212025». https://documentos.cu.ucr.ac.cr/Politicas_Institucionales_2021-2025.pdf

Crenshaw-Williams Kimberlé. 1990. «Mapeo de los márgenes: interseccionalidad, políticas de identidad y violencia contra las mujeres de color». Standford Law Review, 43 (6), 1.241-1.299. http://www.uncuyo.edu.ar/transparencia/upload/crenshaw-kimberlecartografiando-los-margenes-1.pdf

Coller, Xavier. 2005. «Estudio de casos». Madrid: Centro de Investigaciones Sociológicas (CIS).

Domínguez-Domínguez, Alma Rosa y Nilvia Beatriz Vásquez. 2016. «La sobreprotección de los padres en el desarrollo de habilidades adaptativas en personas con discapacidad». Revista de Psicología. https://www.uv.mx/psicologia/files/2016/10/Nilvia.pdf

Donoso, Sebastián y Ernesto Schiefelbein, (2007). «Análisis de los modelos explicativos de retención de estudiantes en la universidad: una visión desde la desigualdad social». Estudios pedagógicos 33(1): 7-27. Valdivia, Chile. https://dx.doi.org/10.4067/S071807052007000100001

Druckemiller, Wendy, Megan Kovacevich, Harold Lawrence/Kleinert y Kathleen SheppardJones. 2015. «Students with intellectual disability in higher education: adult service 
provider perspectives». Intellectual and Developmental Disabilities, 53 (2): 120-128. DOI https://doi.org/10.1352/1934-9556-53.2.120

Durán-Aponte, Emilse y Martín Durán-García. 2015. «Adaptación y validez de un instrumento para la evaluación de docencia universitaria: Escala de Desempeño Docente Institucional (EDDI)». Perspectiva Educacional, Formación de Profesores, 54 (enero): 75-89. http://www.redalyc.org/articulo.oa?id=333333042006

Durán-Aponte, Emilse y María Antonieta Elvira-Valdés. 2015. «Patrones atribucionales y persistencia académica en estudiantes universitarios: validez de la Escala Atribucional de Motivación de Logro General (EAML-G)». Revista Intercontinental De Psicología Y Educación, 17 (2): 201-221. EBSCO Host Academic Search Complete.

Erten, Ozlem. s.f. «Facing Challenges: Experiences of Young Women with Disabilities Attending a Canadian University». Journal of Postsecondary Education and Disability, 24: 101-114. https://files.eric.ed.gov/fulltext/EJ943697.pdf

Espiñeira-Bellón, Eva María, Jesús Miguel Muñoz-Cantero e Isabel Novo-Corti. 2013. «La inclusión de los estudiantes universitarios con discapacidad en las universidades presenciales: actitudes e intención de apoyo por parte de sus compañeros». Estudios Sobre Educación, (24), 103-124. https://hdl.handle.net/10171/29566

Expósito, Francisca, Eva del Río Ferres y Jesús L. Megías, J. 2013. «Gender-based violence against women with visual and physical disabilities». Psicothema, 25 (1): 67-72. DOI https://doi.org/10.7334/psicothema2012.83

Fayez, Simadi e Ibrahim Alqaryouti. (2017). «Students with disabilities’ satisfaction with their universities' services». International Journal of Human Rights in Healthcare, 10(4), 239-247. DOI https://doi.org/10.1108/IJHRH-11-2016-0021

Granada Azcárraga, Maribel, María Pilar Pomés/Correa y Susan Sanhueza-Henríquez. 2013. «Actitud de los profesores hacia la inclusión educativa». Papeles de Trabajo (25): 51-59. https://core.ac.uk/download/pdf/61700917.pdf

Glaser, Barney y Anselm Strauss. 1967. «The discovery of grounded theory: strategies for qualitative research. » New York: Aldine Publishing Company.

Gross Martínez, Marta. 2020. «Cuadernillo de Estrategias Docentes Inclusivas en la Docencia Universitaria». Universidad de Costa Rica, Oficina de Orientación. http://apoyandoteenlau.ucr.ac.cr/serviciosvirtualesoo/2021/CASED/EstrategiasDoce ntesInclusivasCuadernillo.pdf

Heiman, Tali. 2006. «Social support networks, stress, sense of coherence and academic success of university students with learning disabilities». Social Psychology of Education, 9 (4): 461-478. DOI https://doi.org/10.1007/s11218-006-9007-6

Hernández Ruíz, Blanca. 2014. «El concepto de violencia simbólica de Pierre Bourdieu y su aplicación en el ambiente educativo en algunas instituciones educativas bogotanas». Trabajo de grado elaborado como requisito para optar al título de Magíster en 
Educación.https://repository.usta.edu.co/bitstream/handle/11634/14673/2018blanca hernandez.pdf? sequence $=1 \&$ amp;isAllowed $=\mathrm{y}$

Ibáñez, Jesús. 1985. «Análisis sociológico de textos y discursos». Revista internacional de sociología, 43(1): 119.

Karimi, Flora. 2012. «Differential levels of student-related predictors of academic success in the university». The International Journal of Learning, 18 (5). https://www.researchgate.net/publication/288436662_Differential_Levels_of_Stude nt-Internal_Predictors_of_Academic_Success_in_Daystar_University

Kennett, Deborah, Tanya Lewis, Eunice Lund-Lucas, Inez Newbold, Maureen Reed y Carolyn Stallberg. 2009. «The relative effects of university success course and individualized interventions for students with learning disabilities». Higher Education Research \& Development, 28 (4): 385-400. DOI https://doi.org/10.1080/07294360903067013

La Barbera, MariaCaterina. 2016. «Interseccionalidad, un "concepto viajero": orígenes, desarrollo e implementación en la Unión Europea». Interdisciplina, 4 (8): 105-122. http://dx.doi.org/10.22201/ceiich.24485705e.2016.8.54971

Lehtomäki, Elina, Magreth Matonya y Margaret Trotta. 2015. «As Capable as Other Students: Tanzanian Women with Disabilities in Higher Education». International Journal of Disability, Development and Education, 62 (febrero-marzo): 202-214. DOI https://doi.org/10.1080/1034912X.2014.998178

Levitt, Heidi, Michael Bamberg, John Creswell, David Frost, Josselson, Ruthellen, y Carola Suárez-Orozco. 2018. «Normas de informes de artículos de revistas para la investigación cualitativa primaria, cualitativa metaanalítica y de métodos mixtos en psicología: Informe del grupo de trabajo de la Junta de Comunicaciones y Publicaciones de la APA». Psicólogo estadounidense, 73 (1), 26-46. http://dx.doi.org/10.1037/amp0000151

Martínez, Fernando Rey. 2008. «La discriminación múltiple, una realidad antigua, un concepto nuevo». Revista Española de Derecho Constitucional, 28 (84): 251-283. https://dialnet.unirioja.es/servlet/articulo?codigo=2775864

Ferreira, Miguel. 2010. «De la minus-valía a la diversidad funcional: un nuevo marco teóricometodológico/From Handicap to Functional Diversity: a New TheoretialMethodological Framework». Política y sociedad, 47(1): 45-66.

Moriña, Ana. 2016. «Redes de apoyo sociales y académicas de estudiantes con discapacidad que contribuyen a su inclusión en la enseñanza superior». Revista Prisma Social, (16): $32-59$.

http://search.ebscohost.com.ezproxy.sibdi.ucr.ac.cr:2048/login.aspx?direct=true\&db $=$ a 9 h \&AN=123724917\&lang $=$ es \&site=ehost-live \&scope $=$ site

Nicholas, Stephen, Jian Wang y Yaqin Zhong. 2017. «Gender, childhood and adult socioeconomic inequalities in functional disability among Chinese older adults». 
International Journal for Equity in Health, 6 (165). https://equityhealthj.biomedcentral.com/articles/10.1186/s12939-017-0662-3

Ocampo, Aldo. 2013. «Inclusión de estudiantes en situación de discapacidad a la educación $\begin{array}{llllll}\text { superior». } \quad \text { Desafios } & y & \text { oportunidades, } & 6 & \text { (2): } & \text { 227-239. }\end{array}$ http://riberdis.cedd.net/bitstream/handle/11181/5553/Inclusi\%c3\%b3n_de_estudiant es_en_situacion_de_discapacidad.pdf?sequence=1\&amp;rd=0031846690179821

Ocampo González, Aldo. 2016. «Educación Universitaria, interseccionalidad y personas en situación de discapacidad ¿Qué hay más allá de las políticas de afirmación positiva y de reconocimiento?» Experiencias y desafíos sobre Educación Superior Inclusiva.1898. https://dialnet.unirioja.es/servlet/articulo?codigo=5610298

Opini, Batseba. 2012. «Examining the motivations of women students with disabilities' participation in university education in Kenya». Scandinavian Journal of Disability Research, 14 (1): 74- 91. DOI https://doi.org/10.1080/15017419.2010.540853

Pérez-Castro, Judith. 2019. «Entre barreras y facilitadores: las experiencias de los estudiantes universitarios con discapacidad». Sinéctica, 53: 1-22. http://www.scielo.org.mx/pdf/sine/n53/2007-7033-sine-53-00003.pdf

Polo Sánchez, María Tamara y María Dolores López-Justicia. 2016. «Transición Al Empleo De Estudiantes Con Discapacidad: Componentes Para El éxito». Universitas Psychologica 15 (2):273-86. DOI https://doi.org/10.11144/Javeriana.upsy15-2.teus

Ramírez, Marcela. 2011. «Las dimensiones de Accesibilidad en la Universidad de Costa Rica Sede Rodrigo Facio, un Acercamiento desde la Perspectivas de Discapacidad y $\begin{array}{lllll}\text { Género». } & \text { Revista } & \text { Reflexiones. } & 90 & \text { (2): }\end{array}$ http://www.reflexiones.fcs.ucr.ac.cr/images/edicion_90_2/05\%20articulo\%20marce la\%20ramirez.pdf

- 2021. «Las condiciones asociadas a la experiencia de éxito universitario de las mujeres con discapacidad» Tesis de doctorado, Universidad de Costa Rica.

Roca-Cuberes, Carles. 2012. «La percepción de los estudiantes sobre su experiencia de aprendizaje a partir de su dedicación temporal y adquisición de competencias: un estudio sobre la implementación del Grado de Traducción e Interpretación de la Universidad Pompeu Fabra». Revista Complutense de Educación. 24 (2). https://scholar.google.com/citations?view_op=view_citation\&hl=es\&user=z65 2wAAAAJ\&citation_for_view=z-65 2wAAAAJ:IjCSPb-OGe4C

Said-Rücker, Patricia Beatriz. 2011. «Rol de los progenitores en el acceso a la educación superior». Revista de Investigación Educativa, 1 (29): 157-169. https://revistas.um.es/rie/article/view/97811

Saldaña, Magdalena y Omar Barriga. 2010. «Adaptación del modelo de deserción universitaria de Tinto a la Universidad Católica de la Santísima Concepción, Chile». Revista de Ciencias Sociales. 16(4): p. 616-628. http://ve.scielo.org/scielo.php?pid=S1315-95182010000400005\&script=sci_abstract 
Samudio, Edda. 2016. «El acceso de las mujeres a la educación superior. La presencia femenina en la Universidad de Los Andes». Procesos Históricos, 29 (enero-julio): 77-101.https://www.redalyc.org/articulo.oa?id=20044209005

Stake, Robert. 1995. «Investigación con estudios de casos». Ediciones Morata. https://www.uv.mx/rmipe/files/2017/02/Investigacion-con-estudios-de-caso.pdf

Strauss, Anselm, Judith Corbin y Michael Zimmerman. 2002. «Bases de la investigación cualitativa: técnicas y procedimientos para desarrollar la teoría fundamentada». Medellín, Colombia: Universidad de Antioquia.

Taylor, Steven y Robert Bogdan. 1987. «Introducción a los métodos cualitativos de investigación». (Vol. 1). Barcelona: Paidós.

Tinto, Vincent. 1997. «Classrooms as comunnities: Exploring the educational character of student experience». Journal of Higher Education 68(6): 599-623. https://www.uab.edu/2015compliancecertification/IMAGES/SOURCEE148.PDF?id $=40 \mathrm{~d} 5 \mathrm{~d} 239-\mathrm{fd} 27-\mathrm{e} 411-99 \mathrm{c} 8-86539 \mathrm{cf} 2 \mathrm{~d} 30 \mathrm{e}$

- 1975. «Dropout from Higher Education: A theoretical synthesis of recent research». Review of Educational Research 45: 89-125. https://www.jstor.org/stable/1170024

Universidad Estatal a Distancia. 2015. «Lineamientos de política institucional 2015 - 2019». https://www.uned.ac.cr/transparencia/images/documentos/LineamientosPoliticaInsti tucional2015-2019.pdf

Universidad Nacional. 2019. «Política Institucional para el Empleo y la Inclusión de las Personas con Discapacidad». http://documentos.una.ac.cr/handle/unadocs/10274

Yin, Robert. 1994. «Discovering the future of the case study. Method in evaluation research. Evaluation practice» 15 (3): 283-290. 